\title{
Finite Difference Preconditioners for Legendre Based Spectral Element Methods on Elliptic Boundary Value Problems*
}

\author{
Seonhee Kim ${ }^{1}$, Amik St-Cyr², Sang Dong Kim ${ }^{1 \#}$ \\ ${ }^{1}$ Department of Mathematics, Kyungpook National University, Daegu, South Korea \\ ${ }^{2}$ Computation and Modeling, Shell Technology Center Houston (STCH), Houston, USA \\ Email: seonykim@knu.ac.kr, Amik.St-Cyr@shell.com, ${ }^{\#}$ skim@knu.ac.kr
}

Received November 13, 2012; revised April 1, 2013; accepted April 8, 2013

Copyright (C) 2013 Seonhee Kim et al. This is an open access article distributed under the Creative Commons Attribution License, which permits unrestricted use, distribution, and reproduction in any medium, provided the original work is properly cited.

\begin{abstract}
Finite difference type preconditioners for spectral element discretizations based on Legendre-Gauss-Lobatto points are analyzed. The latter is employed for the approximation of uniformly elliptic partial differential problems. In this work, it is shown that the condition number of the resulting preconditioned system is bounded independently of both of the polynomial degrees used in the spectral element method and the element sizes. Several numerical tests verify the $h-p$ independence of the proposed preconditioning.
\end{abstract}

Keywords: Finite Difference Preconditioner; Iterative Method; Spectral Element Method; Elliptic Operator

\section{Introduction}

Linear systems engendered by spectral element discretizations of a simple second-order elliptic boundary value problem have large condition numbers depending on the number of elements $E$ and the polynomial degree $N$ employed. Convergence of iterative solvers thus deteriorates as $E$ and $N$ increases. Regardless of these restrictions, the spectral element method is very popular, accurate and used in many engineering problems. However, it is widely known that an efficient preconditioner is necessary in order to improve the convergence of Krylov subspace methods traditionally used to solve the resulting linear system (see [1-7]). Since the work of [8], it is numerically known that finite difference preconditioning of the spectral element matrix leads to satisfactory results in terms of convergence rates. Multigrid methods are optimal in terms of convergence rate and have linear cost for finite difference problems. The Algebraic multigrid (=: AMG) method can be easily applied to finite difference discretizations of elliptic operators. If it is instead applied directly to high-order discretizations, such as spectral ele-

\footnotetext{
*The third author (corresponding) was supported by basic science research program through the National Research Foundation of Korea (NRF) funded by the ministry of education, science and technology (grant number 2010-0008317) and Kyungpook National University Research Fund 2012.

${ }^{\#}$ Corresponding author.
}

ments, some outstanding issues still need to be addressed. The idea of employing a low-order discretization combined with multigrid as the preconditioner of a high-order problem was investigated in [9] where $P_{1}$ finite elements were employed instead of finite differences. Other efforts concerning the computational cost of $P_{1}$ finite element based two levels additive Schwarz preconditioners can be found in $[10,11]$. In both approaches an intermediate problem for the Laplace equation was constructed using the high-order Legendre-Gauss-Lobatto (=: LGL) nodes. Furthermore, analytic work was performed in [12] for a second-order uniformly elliptic boundary value problem using LGL nodes and also the analytic research based on Chebyshev-Gauss-Lobatto ( $=$ : CGL) nodes was done in [13], in which the various node configurations (LGL and CGL nodes) were employed for the construction of the $P_{1}$ finite element preconditioner. In the 1- and 2-dimensional case, the approach was proven optimal and scalable, respectively.

Thus an efficient and optimal algorithm, with linear cost, for solving problems based on spectral element discretizations, which guarantees the convergence of the overall preconditioning strategy, is readily available. The $P_{1}$ finite element matrix lowers the complexity of the system to invert since the matrices, representing the Laplacian, are tri, penta or hepta diagonal and are easily solvable using the multigrid method. 
Recently Canuto et al. [14,15] have discussed preconditioning with low order finite-elements methods. They show the equivalence between spectral elements and low order finite-elements. The proof is shown for finite-elements and so called numerical integration (NI). In our approach, following motivations found in [4], we propose another preconditioning approach using a simple finite difference scheme based on the LGL nodes in a pointwise approximation perspective. The latter is represented as tensor product matrices, and finite element related properties are employed to prove its optimality. Additionally, it is shown that the proposed preconditioners herein are optimal from the theoretical analysis standpoint and that optimality is confirmed with numerical experiments.

The paper is organized as follows. First, the problem is described in Section 2 and basic definitions are recalled in the same Section 2 also. In the same section, the finite difference preconditioner is introduced. In Section 3, a uniform bound for $\tilde{q}$, the ratio of LGL weights to the distance between the adjacent LGL nodes, is derived which will be used for the $2 D$ case. The optimality results are presented in Sections 4 and 5 with the numerical experiments which support the developed theories. Finally, conclusions are drawn.

\section{Description of the Problem}

Consider the classical boundary value problem of a second-order elliptic operator

$$
A u:=-\nabla \cdot p \nabla u+q u \text { in } \Omega \subset \mathbb{R}^{n}, n=1,2,
$$

where $p \in C^{1}(\bar{\Omega})$ and $q \in C(\bar{\Omega})$ are satisfying

$$
0<p_{0} \leq p \leq p_{1}<\infty, 0 \leq q \leq q_{1}<\infty .
$$

The resulting matrix for the operator $A$, with variable coefficients, discretized using spectral element methods based on LGL nodes is represented as $\hat{A}_{h N}$ and $\hat{A}_{h^{2} N^{2}}$ in one- and two-dimensional case, respectively. Twodimensional matrix $\hat{A}_{h^{2} N^{2}}$ is the result of tensor products using the matrices obtained in the one-dimensional case. We shall consider the preconditioner $\hat{A}_{h}$ and $\hat{A}_{h^{2}}$ for the $1 D$ and $2 D$ case, respectively. Then the problem is to demonstrate the validity of the preconditioners $\hat{L}_{h}$ for $\hat{A}_{h N}$ and $\hat{L}_{h^{2}}$ for $\hat{A}_{h^{2} N^{2}}$, respectively. Moreover, the goal is to show that the preconditioners are optimal in the sense that the condition numbers of the preconditioned systems $\hat{L}_{h}^{-1} \hat{A}_{h N}$ and $\hat{L}_{h^{2}}^{-1} \hat{A}_{h^{2} N^{2}}$ for each case are independent of mesh sizes $h_{j}$ of elements and degrees $N_{k}$ of the piecewise polynomials employed in spectral element discretization.

For the above goal, we will introduce some notations and definitions from now on. Let $\left\{\eta_{k}\right\}_{k=0}^{N}$ and $\left\{\omega_{k}\right\}_{k=0}^{N}$ be the reference LGL points in $[-1,1]$ and quadrature weights associated with the $N^{\text {th }}$-degree Legendre poly- nomial. With dimensionless notations $t$, let us denote $\left\{t_{j}\right\}_{j=0}^{E}$ as the knots in the interval $I=[-1,1]$ such that $-1=: t_{0}<t_{1}<\cdots<t_{E-1}<t_{E}:=1$. For convenience, we denote $N_{j}$ as the degree of Legendre polynomial on each subinterval $I_{j}:=\left[t_{j-1}, t_{j}\right] . \sigma\left(N_{k}\right)$ will be the sum of the degrees of each element up to $k$ : $\sum_{j=1}^{k} N_{j}$. In particular, the notation $\mathfrak{N}:=\sigma\left(N_{E}\right)$ is used when $k=E$ and $\sigma\left(N_{0}\right)=0$. The translated LGL points and corresponding weights from $\left\{\eta_{k}\right\}_{k=0}^{N}$ and $\left\{\omega_{k}\right\}_{k=0}^{N}$ are

$$
\begin{aligned}
\mathcal{G}=\left\{\xi_{k+\sigma\left(N_{j-1}\right)}=: \xi_{j, k}\right\}_{j=1, k=0}^{E, N_{j}} \text { with } \\
\xi_{j, k}=\frac{h_{j}}{2} \eta_{k}+\frac{1}{2}\left(t_{j-1}+t_{j}\right), \quad h_{j}=t_{j}-t_{j-1}
\end{aligned}
$$

and, respectively, $\left\{\rho_{j, k}\right\}_{k=0}^{N_{j}}$ :

$$
\rho_{j, k}:=\left\{\begin{aligned}
& \frac{h_{j}}{2} \omega_{k}, \\
& j=1, k=0 \text { or } j=E, k=N_{E} \\
& j=1,2, \cdots, E, k=1,2, \cdots, N_{j}-1 \\
& \frac{h_{j}}{2} \omega_{N_{j}}+\frac{h_{j+1}}{2} \omega_{0}, \\
& j=1,2, \cdots, E-1, k=N_{j},
\end{aligned}\right.
$$

and $\rho_{j+1,0}:=\rho_{j, N_{j}}$ for $j=1,2, \cdots, E-1$.

For simplicity, all LGL points $\left\{\xi_{\mu}\right\}_{\mu=0}^{\mathfrak{N}}$ are arranged as $-1=: \xi_{0}<\xi_{1}<\cdots<\xi_{\mathfrak{N}}:=1$ and the corresponding LGL weights are ordered as $\left\{\rho_{j, k}\right\}_{j=1, k=0}^{E, N_{j}}=:\left\{\rho_{\mu}\right\}_{\mu=0}^{\mathfrak{N}}$ with $\xi_{E+1,0}:=t_{E}$. Notice that from (2.3) $\xi_{j, N_{j}}=\xi_{j+1,0}$ for $j=1,2, \cdots, E-1$ follows.

Let $\mathcal{P}_{k}$ be the space of all polynomials $p_{k}(t)$ defined on $I=[-1,1]$ whose degrees are less than or equal to $k$ and let $\mathcal{P}_{N}^{h}$ be the subspace of $C(I)$, which consists of piecewise polynomials $p_{N_{j}}(t)$ with support $I_{j}=\left[t_{j-1}, t_{j}\right]$ whose degrees are less than or equal to $N_{j}$. Let $\mathcal{V}_{N}$ be the space of all piecewise Lagrange linear functions $\hat{\psi}_{k}(t)$ with respect to $\left\{\eta_{k}\right\}_{k=0}^{N}$ on $I=[-1,1]$. Define $\mathcal{V}_{N}^{h}$ as the space of all Lagrange piecewise linear functions $\psi_{\mu}(t)$ with respect to $\mathcal{G}$. Let $H_{0}^{1}(I)$ be the standard Sobolev space with zero boundary conditions. Let $\mathcal{P}_{h, N}^{0}:=H_{0}^{1} \cap \mathcal{P}_{N}^{h}$ and $\mathcal{V}_{h, N}^{0}:=H_{0}^{1} \cap \mathcal{V}_{N}^{h}$ be the finite dimensional subspaces of $H_{0}^{1}(I)$ with the basis functions $\left\{\phi_{\mu}\right\}_{\mu=1}^{d}$ and $\left\{\psi_{\nu}\right\}_{\nu=1}^{d}$ respectively, where $d:=\operatorname{dim}\left(\mathcal{P}_{h, N}^{0}\right)=\operatorname{dim}\left(\mathcal{V}_{h, N}^{0}\right)$. To communicate between the space of piecewise linear functions and the space of piecewise polynomial, we use the interpolation operator $\mathcal{I}_{N}^{h}: C(I) \rightarrow \mathcal{P}_{N}^{h}(I)$ such that $\left(\mathcal{I}_{N}^{h} v\right)\left(\xi_{\mu}\right)=v\left(\xi_{\mu}\right)$ for $v \in C(I)$.

For the two-dimensional case, the LGL points are re- 
ordered by horizontal lines or, more precisely, all the points are listed as $\left\{\Xi_{\tilde{\mu}}\right\}_{\tilde{\mu}=1}^{d^{x y}}:=\left\{\left(\xi_{\mu}, \xi_{v}\right)\right\}_{\mu=1, v=1}^{d^{x}, d^{y}}$, where $\tilde{\mu}=\mu+d^{x}(v-1)$ for $\mu=1,2, \cdots, d^{x}, v=1,2, \cdots, d^{y}$. For the two-dimensional high-order and piecewise linear space, let

$$
\left[\mathcal{P}_{h, N}^{0}\right]^{2}:=\mathcal{P}_{h^{x}, N^{x}}^{0} \otimes \mathcal{P}_{h^{y}, N^{y}}^{0},
$$

and

$$
\left[\mathcal{V}_{h, N}^{0}\right]^{2}:=\mathcal{V}_{h^{x}, N^{x}}^{0} \otimes \mathcal{V}_{h^{y}, N^{y}}^{0}
$$

whose basis functions are given by tensor products of one-dimensional piecewise Lagrange polynomials and linear functions, respectively. Let $d^{x y}:=\operatorname{dim}\left(\left[\mathcal{P}_{h, N}^{0}\right]^{2}\right)$. Note that $d^{x y}=d^{x} d^{y}$, where $d^{t}:=\operatorname{dim}\left(\mathcal{P}_{h^{t}, N^{t}}^{0}\right), t=x$ or $y$.

To provide the preconditioner, using the global LGL points $\left\{\xi_{\mu}\right\}$, we define the operator $B_{h}$ on $\mathcal{V}_{h, N}^{0}$

$$
\left[B_{h} u\right]_{\mu}=\left[-\frac{u_{\mu-1}}{s_{\mu-1}}+\left(\frac{1}{s_{\mu-1}}+\frac{1}{s_{\mu}}\right) u_{\mu}-\frac{u_{\mu+1}}{s_{\mu}}\right],
$$

where $s_{\mu}:=\xi_{\mu+1}-\xi_{\mu}, u_{\mu}:=u\left(\xi_{\mu}\right)$.

With this operator and components $\alpha>0, \beta \geq 0$, we define the one-dimensional finite difference operator $L_{h}$ as following:

$$
\left[L_{h} u\right]_{\mu}=\alpha\left[B_{h} u\right]_{\mu}+\beta \tilde{s}_{\mu} u_{\mu},
$$

where $\tilde{s}_{\mu}:=s_{\mu}+s_{\mu-1}=\xi_{\mu+1}-\xi_{\mu-1}$.

The two-dimensional finite difference operator $L_{h^{2}}$ is therefore defined as

$$
\begin{aligned}
{\left[L_{h^{2}} u\right]_{\mu, v}=} & \tilde{s}_{v}^{y}\left(\alpha\left[B_{h}^{x} u\right]_{\mu, v}+\beta \tilde{s}_{\mu}^{x} u_{\mu, v}\right) \\
& +\tilde{s}_{\mu}^{x}\left(\alpha\left[B_{h}^{y} u\right]_{\mu, v}+\beta \tilde{s}_{v}^{y} u_{\mu, v}\right),
\end{aligned}
$$

where $\left[B_{h}^{t} u\right]_{\mu, v}$ is the $2 D$ version of (2.5) for $t=x$ or $y$, precisely,

$$
\begin{gathered}
{\left[B_{h}^{x} u\right]_{\mu, v}:=-\frac{u_{\mu-1, v}}{s_{\mu-1}^{X}}+\left(\frac{1}{s_{\mu-1}^{x}}+\frac{1}{s_{\mu}^{x}}\right) u_{\mu, v}-\frac{u_{\mu+1, v}}{s_{\mu}^{x}},} \\
{\left[B_{h}^{y} u\right]_{\mu, v}:=-\frac{u_{\mu, v-1}}{s_{v-1}^{y}}+\left(\frac{1}{s_{v-1}^{y}}+\frac{1}{s_{v}^{y}}\right) u_{\mu, v}-\frac{u_{\mu, v+1}}{s_{v}^{y}},}
\end{gathered}
$$

and $s_{\gamma}^{t}=\xi_{\gamma+1}^{t}-\xi_{\gamma}^{t}, \quad \tilde{s}_{\gamma}^{t}=\xi_{\gamma+1}^{t}-\xi_{\gamma-1}^{t}$ for $t=x$ or $y$ and $u_{\mu, v}:=u\left(\xi_{\mu}, \xi_{v}\right)$.

Finally, the notation $a \sim b$ for any two real quantities $a$ and $b$ is a shorthand notation corresponding to the existence of two positive constants $c$ and $C$, which do not depend on the mesh sizes and the degrees of polynomials such that $0<c \leq \frac{a}{b} \leq C<\infty$. The notation $(U, V)$ stands for $\sum u_{i} v_{i}$ for any two vectors $U=\left(u_{1}, \cdots, u_{d}\right)^{\mathrm{T}}$ and $V=\left(v_{1}, \cdots, v_{d}\right)^{\mathrm{T}}$ where the superscript $\mathrm{T}$ denotes the transpose of a vector or matrix. The standard Sobolev space $H^{1}$ and $L^{2}$ will be used. And we will use $\|\cdot\|_{1},||_{1}$ and $\|\cdot\|_{0}$ as the standard Sobolev $H^{1}$-norm, $H^{1}$-seminorm and the usual $L^{2}$-norm, respectively.

\section{Basic Estimates}

We begin by recalling the relations between the distances of LGL nodes and the LGL weights in the reference element $[-1,1]$ found in [6].

Lemma 3.1. With LGL nodes $\eta_{k}$ and LGL weights $\omega_{k}$, let

$$
\begin{aligned}
q_{0} & :=\frac{2 \omega_{0}}{\eta_{1}-\eta_{0}}, \quad q_{N}:=\frac{2 \omega_{N}}{\eta_{N}-\eta_{N-1}}, \\
q_{k} & :=\frac{2 \omega_{k}}{\eta_{k+1}-\eta_{k-1}}, \quad k=1, \cdots, N-1 .
\end{aligned}
$$

Then the $q_{k}$ are uniformly bounded for all $k=0,1, \cdots, N$. In particular,

$$
0<\alpha_{1} \leq q_{0} \leq \alpha_{2} \text { and } 0<\beta_{1} \leq q_{N} \leq \beta_{2},
$$

where $\alpha_{i}, \beta_{i}(i=1,2)$ are constants independent of the polynomial degree $N$.

Proof. See Lemma 3.1 in [6].

Since the goal is to develop preconditioners on spectral elements considering different polynomial degrees on each elements of which sizes are not identical, we need an advanced version or $\mathcal{G}$-version of Lemma 3.1. Hence the modifications of (3.1)-(3.2) for $\left\{\tilde{q}_{\mu}\right\}_{\mu=0}^{\mathfrak{N}}:=\left\{q_{j, k}\right\}_{j=1, k=0}^{E, N_{j}}$ are required, where $\mu=k+\sigma\left(N_{j-1}\right)$.

First, let $\tilde{q}_{0}, \quad \tilde{q}_{\mathfrak{N}}$ and $\tilde{q}_{\mu} \quad(\mu=1,2, \cdots, \mathfrak{N}-1)$ be defined as

$$
\tilde{q}_{0}:=\frac{2 \rho_{0}}{\xi_{1}-\xi_{0}}, \quad \tilde{q}_{\mathfrak{N}}:=\frac{2 \rho_{\mathfrak{N}}}{\xi_{\mathfrak{N}}-\xi_{\mathfrak{N}-1}},
$$

and

$$
\tilde{q}_{\mu}:=\frac{2 \rho_{\mu}}{\xi_{\mu+1}-\xi_{\mu-1}} \text { for } \mu=1,2, \cdots, \mathfrak{N}-1 .
$$

Lemma 3.2. The $\tilde{q}_{\mu}$ given in (3.4)-(3.5) are bounded for all $\mu=0,1, \cdots, \mathfrak{N}$ independently of both degrees $N_{k}$ of polynomials and the mesh sizes $h_{j}$ of each element.

Proof. Since the LGL nodes in each subinterval are defined by an affine map transformation from the ones in reference interval $[-1,1]$, they depend on the mesh sizes of each element thus they are distinguished as follows 


$$
\tilde{q}_{\mu}=q_{j, k}=\left\{\begin{array}{c}
\frac{2 \rho_{1,0}}{\xi_{1,1}-\xi_{1,0}}\left(=\tilde{q}_{0}\right), \\
j=1, k=0, \\
\frac{2 \rho_{E, N_{E}}}{\xi_{E, N_{E}}-\xi_{E, N_{E}-1}}\left(=\tilde{q}_{\mathfrak{N}}\right), \\
j=E, k=N_{E}, \\
\frac{2 \rho_{j, k}}{\xi_{j, k+1}-\xi_{j, k-1}}, \\
j=1,2, \cdots, E, k=1,2, \cdots, N_{j}-1, \\
2 \rho_{j, N_{j}} \\
\frac{\left(\xi_{j, N_{j}}-\xi_{j, N_{j}-1}\right)+\left(\xi_{j+1,1}-\xi_{j+1,0}\right)}{j=1,2, \cdots, E-1, k=N_{j} .}
\end{array}\right.
$$

Since the first and second cases of (3.6) are

$$
\tilde{q}_{0}=\frac{2 \frac{h_{1}}{2} \omega_{0}}{\frac{h_{1}}{2}\left(\eta_{1}-\eta_{0}\right)}=q_{0}
$$

and

$$
\tilde{q}_{\mathfrak{N}}=\frac{2 \frac{h_{E}}{2} \omega_{N_{E}}}{\frac{h_{E}}{2}\left(\eta_{N_{E}}-\eta_{N_{E}-1}\right)}=q_{N_{E}},
$$

it is clear that $\tilde{q}_{0}, \tilde{q}_{\mathfrak{N}}$ are uniformly bounded by Lemma 3.1.

For the case $j=1,2, \cdots, E, k=1,2, \cdots, N_{j}-1$, it can be easily shown that

$$
q_{j, k}=\frac{2 \rho_{j, k}}{\xi_{j, k+1}-\xi_{j, k-1}}=\frac{2 \frac{h_{j}}{2} \omega_{k}}{\frac{h_{j}}{2}\left(\eta_{k+1}-\eta_{k-1}\right)}=q_{k} .
$$

Lastly for $j=1,2, \cdots, E-1, \quad k=N_{j}$, note that

$$
q_{j, N_{j}}=\frac{2\left(\frac{h_{j}}{2} \omega_{N_{j}}+\frac{h_{j+1}}{2} \omega_{0}\right)}{\frac{h_{j}}{2}\left(\eta_{N_{j}}-\eta_{N_{j-1}}\right)+\frac{h_{j+1}}{2}\left(\eta_{1}-\eta_{0}\right)} .
$$

Using (3.3), it comes

$$
\left(\eta_{1}-\eta_{0}\right) \alpha_{1} \leq \omega_{0} \leq\left(\eta_{1}-\eta_{0}\right) \beta_{1}
$$

and

$$
\left(\eta_{N_{j}}-\eta_{N_{j-1}}\right) \alpha_{2} \leq \omega_{N_{j}} \leq\left(\eta_{N_{j}}-\eta_{N_{j-1}}\right) \beta_{2},
$$

where $\alpha_{i}, \beta_{i}(i=1,2)$ are the absolute positive constants that appear in Lemma 3.1. Therefore,

$$
2 \min \left(\alpha_{1}, \alpha_{2}\right) \leq q_{j, N_{j}} \leq 2 \max \left(\beta_{1}, \beta_{2}\right)
$$

which completes the proof.

Define the two matrices $W$ and $H$, which are made up of, respectively, the quadrature weights and the distances between the LGL points:

$$
\begin{aligned}
W & :=\operatorname{diag}\left(\rho_{\mu}\right), \\
H & :=\operatorname{diag}\left(\xi_{\mu+1}-\xi_{\mu-1}\right) \\
& =\operatorname{diag}\left(s_{\mu}+s_{\mu-1}\right)=\operatorname{diag}\left(\tilde{s}_{\mu}\right),
\end{aligned}
$$

where $\mu=1,2, \cdots, d$ and $\xi_{0}=\xi_{d+1}=0$.

Notice that the quantities $\xi_{\mu+1}-\xi_{\mu-1}$ and $\rho_{\mu}$ are positive for all $\mu=1,2, \cdots, d$. By Lemma 3.2, $\tilde{q}_{\mu}$ defined in (3.5) are uniformly bounded. Thus we have the following corollary:

Corollary 3.3. For $U=\left(u_{1}, u_{2}, \cdots, u_{d}\right)^{\mathrm{T}}$, it follows that

$$
(H U, U) \sim(W U, U) .
$$

The next result is a clear consequence but we write down for convenience.

Lemma 3.4. For any diagonal matrix $S$ with nonnegative entries whose size is the same as $W$ (see definition in (3.7)),

$$
((S W) U, U) \sim(W U, U),
$$

where the equivalence depends on the minimum and maximum entries of $S$.

The matrix $W$ will be replaced by $W \otimes W$ for twodimensional problems.

Proposition 3.5. Let E, F be symmetric and positive definite matrices such that

$$
(E V, V) \sim(F V, V)
$$

for any nonzero vector $V$. Then for any symmetric and positive definite matrix $G$, we have

$$
\begin{aligned}
& ((G \otimes E) V, V) \sim((G \otimes F) V, V), \\
& ((E \otimes G) V, V) \sim((F \otimes G) V, V) .
\end{aligned}
$$

Proof. Since all the matrices are symmetric and positive definite, it is enough to discuss (3.9) in terms of eigenvalues.

Consider the eigenvalue problem

$$
E U_{\mu}=\kappa_{\mu} F U_{\mu} .
$$

It has a complete set of eigenpairs $\left(U_{\mu}, \kappa_{\mu}\right)$, $\mu=1,2, \cdots, d^{x}$. Let

$$
Z_{\mu v}=\mathbf{1}_{v} \otimes U_{\mu}, \Lambda_{\mu v}=1_{v} \kappa_{\mu}\left(=\kappa_{\mu}\right),
$$

where $\mathbf{1}$ is a vector consisting of element 1 with length $d^{y}$.

\section{Since}




$$
\begin{aligned}
(G \otimes E) Z_{\mu v} & =(G \otimes E)\left(\mathbf{1}_{v} \otimes U_{\mu}\right)=G \mathbf{1}_{v} \otimes E U_{\mu} \\
& =G \mathbf{1}_{v} \otimes \kappa_{\mu} F U_{\mu}=\kappa_{\mu}\left(G \mathbf{1}_{v} \otimes F U_{\mu}\right) \\
& =\kappa_{\mu}(G \otimes F)\left(\mathbf{1}_{v} \otimes U_{\mu}\right) \\
& =1_{v} \kappa_{\mu}(G \otimes F)\left(\mathbf{1}_{v} \otimes U_{\mu}\right) \\
& =\Lambda_{\mu v}(G \otimes F) Z_{\mu v},
\end{aligned}
$$

the vectors and eigenvalues in (3.10) are complete sets of eigenvectors and eigenvalues of the eigenvalue problem

$$
(G \otimes E) V=\Lambda(G \otimes F) V .
$$

From (3.8), since $\kappa_{\mu}$ are positive, bounded and independent of $h_{j}^{x}, N_{k}^{x}$, so are $\Lambda_{\mu v}$. Therefore it follows

$((G \otimes E) V, V) \sim((G \otimes F) V, V)$ for any vector $V$.

By the same reasoning, it follows

$$
((E \otimes G) V, V) \sim((F \otimes G) V, V) .
$$

Finally, the known results stated in Theorem 3.3 and 3.5 from [12] are recalled here for completeness.

Theorem 3.6. It follows that for all $u \in \mathcal{V}_{N}^{h}$,

$$
\left|\mathcal{I}_{N}^{h} u\right|_{1} \sim|u|_{1} \text { and }\left\|\mathcal{I}_{N}^{h} u\right\|_{0} \sim\|u\|_{0} \text {. }
$$

\section{One-Dimensional Case}

Before going ahead, suppose that $p \in C^{1}(\bar{\Omega})$ and $q \in C(\bar{\Omega})$ satisfy $0<\hat{p}_{l} \leq p(x) \leq \hat{p}_{u}<\infty$ and $0 \leq \hat{q}_{l} \leq q(x) \leq \hat{q}_{u}<\infty$, where $\hat{p}_{l}, \quad \hat{p}_{u}, \quad \hat{q}_{l}$ and $\hat{q}_{u}$ are constants. Now consider the following elliptic boundary value problem with zero boundary condition:

$$
A u:=-\left(p(x) u^{\prime}(x)\right)^{\prime}+q(x) u(x) \text { in } \Omega=(-1,1) .
$$

Expanding $p(x)$ and $q(x)$ in (4.1) on the space $\mathcal{P}_{h, N}^{0}$ as $p(x)=\sum_{\mu=1}^{d} p_{\mu} \phi_{\mu}$ and $q(x)=\sum_{\mu=1}^{d} q_{\mu} \phi_{\mu}$, the matrix representation of the operator $A$ in (4.1) by the spectral element discretization based on LGL points is now given by

$$
\hat{A}_{h N}:=D^{\mathrm{T}}(P W) D+Q W,
$$

where $P=\operatorname{diag}\left(p_{1}, \cdots, p_{d}\right), Q=\operatorname{diag}\left(q_{1}, \cdots, q_{d}\right), D$ is the differentiation matrix defined as $D_{\mu \nu}:=\phi_{\nu}^{\prime}\left(\xi_{\mu}\right)$ and $W$ is the matrix defined in (3.7).

Since $P$ and $Q$ are the diagonal matrices with nonnegative elements, by Lemma 3.4 we have for any vector U,

$$
(\tilde{K} U, U):=\left(\left(D^{\mathrm{T}}(P W) D\right) U, U\right) \sim\left(\left(D^{\mathrm{T}} W D\right) U, U\right)
$$

and

$$
(Q W U, U) \sim(W U, U) .
$$

More precisely, it follows that

$$
\begin{gathered}
\hat{p}_{l}\left(\left(D^{\mathrm{T}} W D\right) U, U\right) \leq(\tilde{K} U, U) \leq \hat{p}_{u}\left(\left(D^{\mathrm{T}} W D\right) U, U\right) \\
\hat{q}_{l}(W U, U) \leq(Q W U, U) \leq \hat{q}_{u}(W U, U)
\end{gathered}
$$

Besides, we can see that for

$$
\begin{gathered}
u_{N}(t)=\sum_{\mu=1}^{d} u_{\mu} \phi_{\mu}(t) \in \mathcal{P}_{h, N}^{0}, \\
\left(\left(D^{\mathrm{T}} W D\right) U, U\right) \sim\left|u_{N}\right|_{1}^{2}, \\
(W U, U) \sim\left\|u_{N}\right\|_{0}^{2},
\end{gathered}
$$

where $U=\left(u_{1}, u_{2}, \cdots, u_{d}\right)^{\mathrm{T}}$.

Let $\hat{B}_{h}$ be the matrix representation of $B_{h}$, which is defined in (2.5). For $u(t)=\sum_{\mu=1}^{d} u_{\mu} \psi_{\mu}(t) \in \mathcal{V}_{h, N}^{0}$, the easy calculation leads to

$$
C_{0}|u|_{1}^{2} \leq\left(\hat{B}_{h} U, U\right) \leq C_{1}|u|_{1}^{2},
$$

where $U=\left(u_{1}, u_{2}, \cdots, u_{d}\right)^{\mathrm{T}}$.

Note that the constants such as $C_{0}, C_{1}$ appeared in this and next sections are generic positive constants, independent of the number of elements $E_{i}$ and the degree $N_{j}$ of polynomials applied to spectral discretization.

Now to provide a finite difference type preconditioner, consider the bilinear form $l_{N}^{(0)}(u, v)$ with a positive constant $\alpha$, defined on the space $\mathcal{V}_{h, N}^{0} \times \mathcal{V}_{h, N}^{0}$

$$
l_{N}^{(0)}(u, v)=\alpha \sum_{\mu=1}^{d}\left[B_{h} u\right]_{\mu} v_{\mu},
$$

where $u_{\mu}=u\left(\xi_{\mu}\right)$. This induces the matrix $\hat{L}_{h, 0}$ :

$$
\begin{gathered}
l_{N}^{(0)}(u, v)=\left(\hat{L}_{h, 0} U, V\right), \\
U=\left(u_{1}, \cdots, u_{d}\right)^{\mathrm{T}}, V=\left(v_{1}, \cdots, v_{d}\right)^{\mathrm{T}} .
\end{gathered}
$$

Thus it comes immediately

$$
\hat{L}_{h, 0}=\alpha \hat{B}_{h} \text {. }
$$

Lemma 4.1. For any vector $U=\left(u_{1}, u_{2}, \cdots, u_{d}\right)^{\mathrm{T}}$, it follows that

$$
0<C_{0} \frac{\hat{p}_{l}}{\alpha} \leq \frac{\left(\hat{A}_{h N} U, U\right)}{\left(\hat{L}_{h, 0} U, U\right)} \leq C_{1} \frac{\max \left(\hat{p}_{u}, \hat{q}_{u}\right)}{\alpha} .
$$

Proof. Note that $u \in \mathcal{V}_{h, N}^{0}$ can be expressed as $u(t)=\sum_{\mu=1}^{d} u_{\mu} \psi_{\mu}(t)$, so that its piecewise polynomial interpolation becomes $\left(\mathcal{I}_{N}^{h} u\right)(t)=\sum_{\mu=1}^{d} u_{\mu} \phi_{\mu}(t) \in \mathcal{P}_{h, N}^{0}$.

Since $(\tilde{K} U, U) \leq\left(\hat{A}_{h N} U, U\right)=(\tilde{K} U, U)+(Q W U, U)$, it follows from (4.3)-(4.5), that

$$
\begin{aligned}
\hat{p}_{l}\left|\mathcal{I}_{N}^{h} u\right|_{1}^{2} & \leq\left(\hat{A}_{h N} U, U\right) \leq c \max \left(\hat{p}_{u}, \hat{q}_{u}\right)\left\|\mathcal{I}_{N}^{h} u\right\|_{1}^{2} \\
& \leq C \max \left(\hat{p}_{u}, \hat{q}_{u}\right)\left|\mathcal{I}_{N}^{h} u\right|_{1}^{2} .
\end{aligned}
$$

The last inequality is due to Poincare's inequality. 
Therefore, (4.7), (4.6) and Theorem 3.6 complete the proof.

Remark 1. From the above lemma, one may notice that the upper bound for the condition numbers of the matrix $\hat{L}_{h, 0}^{-1} \hat{A}_{h N}$ does not depend on the mesh size $h_{j}$ of an element and the degree $N_{i}$ of a polynomial. Further, it does not depend on $\alpha$. Hence, even if (4.1) has coefficient functions $p(x)$ and $q(x)$, it is enough to take the preconditioner $\hat{L}_{h, 0}=\hat{B}_{h}$ with $\alpha=1$ in (4.7).

Next, we consider another bilinear form $l_{N}(u, v)$ on the space $\mathcal{V}_{h, N}^{0} \times \mathcal{V}_{h, N}^{0}$ with a constant $\beta \geq 0$

$$
l_{N}(u, v):=l_{N}^{(0)}(u, v)+\beta \sum_{\mu=1}^{d} \tilde{s}_{\mu} u_{\mu} v_{\mu},
$$

where $u_{\mu}=u\left(\xi_{\mu}\right)$ and $\tilde{s}_{\mu}=\xi_{\mu+1}-\xi_{\mu-1}$. The matrix representation $\hat{L}_{h}$ corresponding to $l_{N}(\cdot, \cdot)$ is

$$
\begin{gathered}
l_{N}(u, v)=\left(\hat{L}_{h} U, V\right), \\
U=\left(u_{1}, \cdots, u_{d}\right)^{\mathrm{T}}, V=\left(v_{1}, \cdots, v_{d}\right)^{\mathrm{T}},
\end{gathered}
$$

where

$$
\hat{L}_{h}:=\alpha \hat{B}_{h}+\beta H .
$$

Now the goal is to analyze the validity of the matrix operator $\hat{L}_{h}$ for the preconditioner to $\hat{A}_{h N}$.

Theorem 4.2. For any vector $U=\left(u_{1}, u_{2}, \cdots, u_{d}\right)^{\mathrm{T}}$, it follows that

$$
\left(\hat{A}_{h N} U, U\right) \sim\left(\hat{L}_{h} U, U\right),
$$

where the equivalence depends only on $\hat{p}_{u}, \hat{p}_{l}, \hat{q}_{u}$, $\hat{q}_{l}, \alpha$ and $\beta$.

Proof. For $u \in \mathcal{V}_{h, N}^{0}$ such that $u(t)=\sum_{\mu=1}^{d} u_{\mu} \psi_{\mu}(t)$, its piecewise polynomial interpolation is

$$
\begin{aligned}
\left(\mathcal{I}_{N}^{h} u\right)(t)=\sum_{\mu=1}^{d} u_{\mu} \phi_{\mu}(t) & \in \mathcal{I}_{h, N}^{0} . \text { Using (4.3)-(4.5), we get } \\
c \min \left(\hat{p}_{l}, \hat{q}_{l}\right)\left\|\mathcal{I}_{N}^{h} u\right\|_{1}^{2} & \leq\left(\hat{A}_{h N} U, U\right) \\
& \leq C \max \left(\hat{p}_{u}, \hat{q}_{u}\right)\left\|\mathcal{I}_{N}^{h} u\right\|_{1}^{2} .
\end{aligned}
$$

On the other hand, applying Corollary 3.3, (4.5) and Theorem 3.6 we have

$$
(H U, U) \sim\|u\|_{0}^{2} \text { for } u \in \mathcal{V}_{h, N}^{0}
$$

so that

$$
c^{\prime} \min (\alpha, \beta)\|u\|_{1}^{2} \leq\left(\hat{L}_{h} U, U\right) \leq C^{\prime} \max (\alpha, \beta)\|u\|_{1}^{2} .
$$

Hence, using Theorem 3.6 again, we have

$$
C_{0} \frac{\min \left(\hat{p}_{l}, \hat{q}_{l}\right)}{\max (\alpha, \beta)} \leq \frac{\left(\hat{A}_{h N} U, U\right)}{\left(\hat{L}_{h} U, U\right)} \leq C_{1} \frac{\max \left(\hat{p}_{u}, \hat{q}_{u}\right)}{\min (\alpha, \beta)} .
$$

To guarantee the positivity of the lower bound, if $\hat{q}_{l}=0$, we will take $\beta=0$. Applying Poincare's inequality with (4.9) and (4.6), we have
$(H U, U) \leq C\left(\hat{B}_{h} U, U\right)$ for some positive constant $C$. Therefore, using (4.3)-(4.5) and Theorem 3.6, we get

$$
\begin{aligned}
c_{0} \hat{p}_{l}\left(\hat{B}_{h} U, U\right) & \leq(\tilde{K} U, U)+(Q W U, U) \\
& \leq C_{1} \max \left(\hat{p}_{u}, \hat{q}_{u}\right)\left(\hat{B}_{h} U, U\right)
\end{aligned}
$$

so that

$$
C_{0} \frac{\hat{p}_{l}}{\alpha} \leq \frac{\left(\hat{A}_{h N} U, U\right)}{\left(\hat{L}_{h} U, U\right)} \leq C_{1} \frac{\max \left(\hat{p}_{u}, \hat{q}_{u}\right)}{\alpha},
$$

which leads to the conclusion.

The latter, combined with the min-max theorem, yields the next result.

Corollary 4.3. The eigenvalues $\left\{\lambda_{\mu}\right\}_{\mu=1}^{d}$ of $\hat{L}_{h}^{-1} \hat{A}_{h N}$ are all positive real and bounded above and below. The bounds are independent of the mesh sizes $h_{j}$ and the degrees of the polynomials $N_{k}$. That is, there are absolute constants $C_{0}$ and $C_{1}$ such that

$$
0<C_{0} \leq \lambda_{\mu} \leq C_{1}<\infty .
$$

Remark 2. Theorem 4.2 reveals that the condition numbers of $\hat{L}_{h}^{-1} \hat{A}_{h N}$ are bounded above by $\frac{\max \left(\hat{p}_{u}, \hat{q}_{u}\right)}{\min \left(\hat{p}_{l}, \hat{q}_{l}\right)} \frac{\max (\alpha, \beta)}{\min (\alpha, \beta)}$ or $\frac{\max \left(\hat{p}_{u}, \hat{q}_{u}\right)}{\hat{p}_{l}}$. Hence one may notice that the condition numbers do not depend on the degrees of polynomials $N_{k}$ and the mesh sizes $h_{j}$.

According to Remark 2, we may summarize the behavior of the condition numbers of $\hat{L}_{h}^{-1} \hat{A}_{h N}$ in the following corollary.

Corollary 4.4. The upper bound of the the condition numbers of $\hat{L}_{h}^{-1} \hat{A}_{h N}$ behaves like $\frac{\hat{p}_{u}}{\hat{p}_{l}}, \frac{\hat{q}_{u}}{\hat{p}_{l}}, \frac{\hat{p}_{u}}{\hat{q}_{l}}$ or $\frac{\hat{q}_{u}}{\hat{q}_{l}}$.

We will investigate the efficiency of the preconditioners $\hat{L}_{h, 0}=\alpha \hat{B}_{h}$ and $\hat{L}_{h}=\alpha \hat{B}_{h}+\beta H$ for several problems with constant and variable coefficients. Moreover, for a variable coefficients problem, we will compare the developed preconditioners with the $P_{1}$ finite element preconditioner (see [12]) in terms of iteration numbers using the preconditioned conjugate gradient method (=: PCG).

Example 1. Consider the operator with constant coefficients:

$$
A u:=-p u^{\prime \prime}+q u \text { in }(-1,1)
$$

with homogeneous Dirichlet boundary conditions, where $p>0$ and $q \geq 0$ are constants. Note that the spectral element discretized matrix corresponding to (4.2) becomes $\hat{A}_{h N}=p D^{\mathrm{T}} W D+q W$. Since the condition numbers are dependent on the ratios $q / p$ and $\alpha$ by the 
definition, we take $\alpha=1$ and focus mainly on the role of $\beta$, where $\hat{L}_{h}=\hat{B}_{h}+\beta H$.

First, it is computed with $\hat{L}_{h, 0}=\hat{B}_{h}$. Figure 1 is shown to be ineffective for large values $q / p$, but we can see that $\hat{L}_{h, 0}=\hat{B}_{h}$ is effective for $q / p$-value equal or less than 1 (see Table 1).

Second, to show that the preconditioning work is not independent of the polynomial degrees $N$ and the numbers of element $E$, applied in spectral element method, it is tested for the cases with $N=4,8,12, \cdots, 32$ and $E=1,2,4, \cdots, 32$. For the values $q / p=10^{-3}, 10^{-2}, \cdots, 10^{3}$, the condition numbers of $\hat{A}_{h N}=p D^{\mathrm{T}} W D+q W$ go up to approximately $4.5 \times 10^{6}$ when $N=E=32$, but ones of the matrices preconditioned by $\hat{L}_{h, 0}=\hat{B}_{h}$ are bounded above by approximately 4.5 , independently of $N, E$ (see Figure 2).

Third, We investigated the condition numbers of the matrices by preconditioned by $\hat{L}_{h}=\hat{B}_{h}+\beta H$ with several $\beta$-values for each $q / p$-value (see Figure 3). When $\beta$ is taken to be equal to $q / p$-value or $\max \{1, q / p\}$, it gives good preconditioning results (see Figure 4).

Example 2. Now we consider the operator with the variable coefficients:

$$
A u:=-\left(p u^{\prime}\right)^{\prime}+q u \text { in }(-1,1)
$$

with homogeneous Dirichlet boundary conditions, where $p(x)=\hat{p}\left(x^{2}+1\right)$ and $q(x)=\hat{q} \cos x$, where $\hat{p}>0$ and $\hat{q} \geq 0$ are constants. In this case, the spectral element discretization yield the matrix

$\hat{A}_{h N}=\hat{p} D^{\mathrm{T}}(P W) D+\hat{q} Q W$, where $P$ and $Q$ are the matrices of $x^{2}+1$ and $\cos x$ represented by spectral

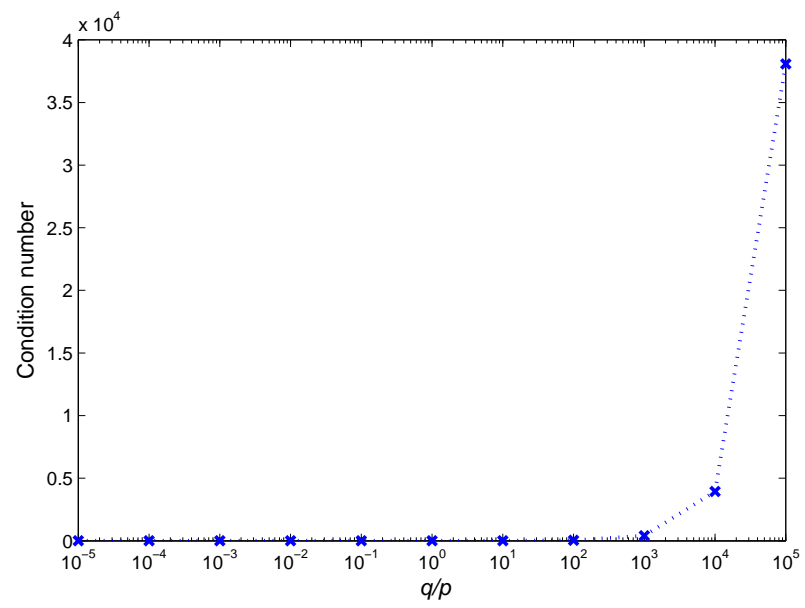

Figure 1. Condition numbers of $\hat{\boldsymbol{L}}_{h, 0}^{-1} \hat{A}_{h N}$, where $\hat{\boldsymbol{L}}_{h, 0}=\hat{B}_{h}$.

Table 1. Condition numbers of $\hat{L}_{h, 0}^{-1} \hat{A}_{h N}$, where $\hat{L}_{h, 0}=\hat{B}_{h}$.

\begin{tabular}{ccccccc}
\hline$q / p$ & $10^{-5}$ & $10^{-4}$ & $10^{-3}$ & $10^{-2}$ & $10^{-1}$ & $10^{0}$ \\
\hline$\kappa$ & $2: 3239$ & $2: 3238$ & $2: 3237$ & $2: 3236$ & $2: 3230$ & $5: 0419$ \\
\hline
\end{tabular}
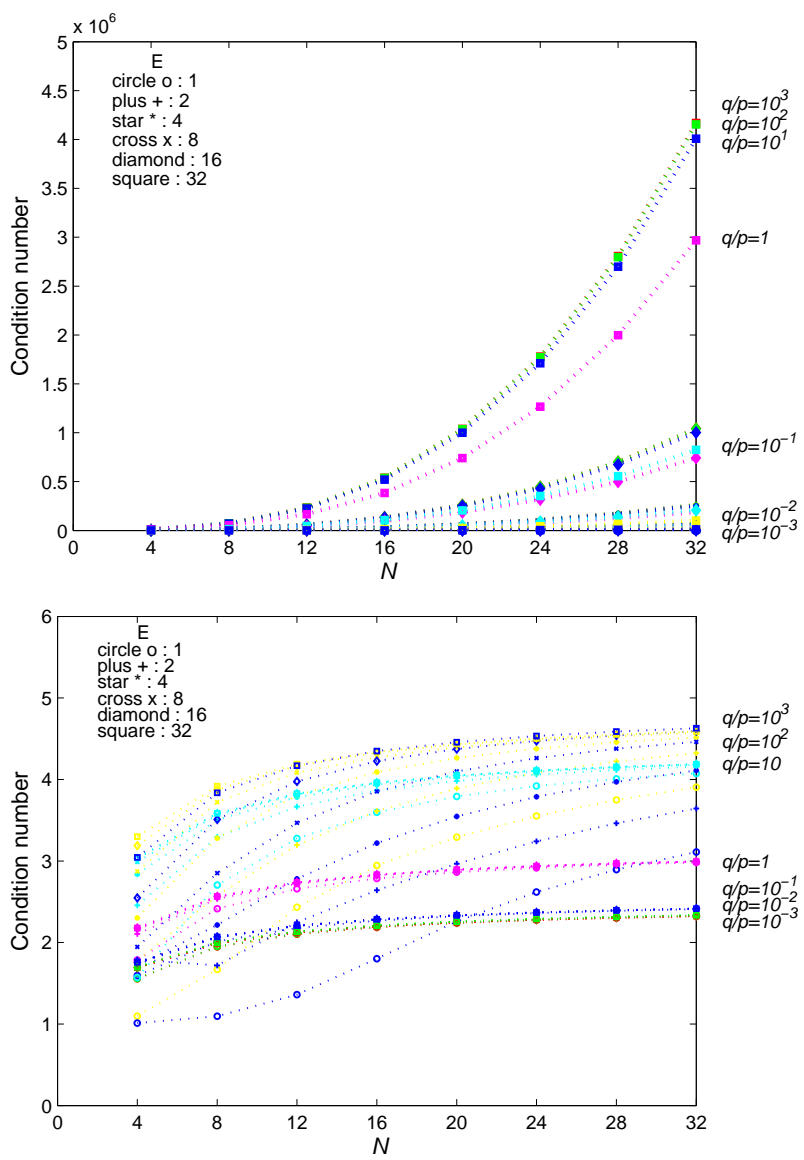

Figure 2. Condition numbers of $\hat{A}_{h N}$ and $\hat{L}_{h, 0}^{-1} \hat{A}_{h N}$, where $\hat{\boldsymbol{L}}_{h, 0}=\hat{B}_{h}$.

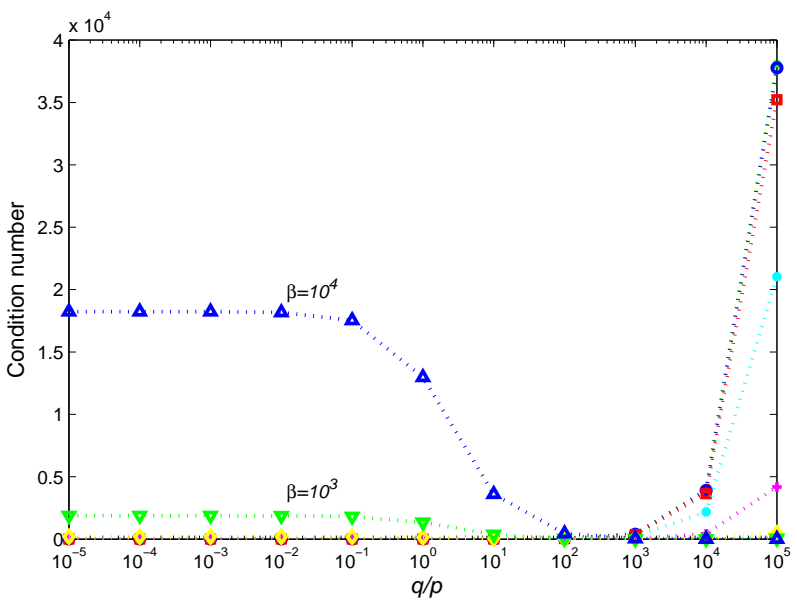

Figure 3. Behaviors of $\kappa\left(\hat{L}_{h}^{-1} \hat{A}_{h N}\right)$, where $\hat{L}_{h}=\hat{B}_{h}+\beta H$.

element discretization, respectively. Figure 5 shows that $\beta=\hat{q} / 2 \hat{p}$ or $\beta=\max \{1, \hat{q} / 2 \hat{p}\}$ give the good preconditioning results.

Example 3. For the variable coefficients problem of Example 2 with $p(x)=x^{2}+1$ and $q(x)=\cos x$ : 


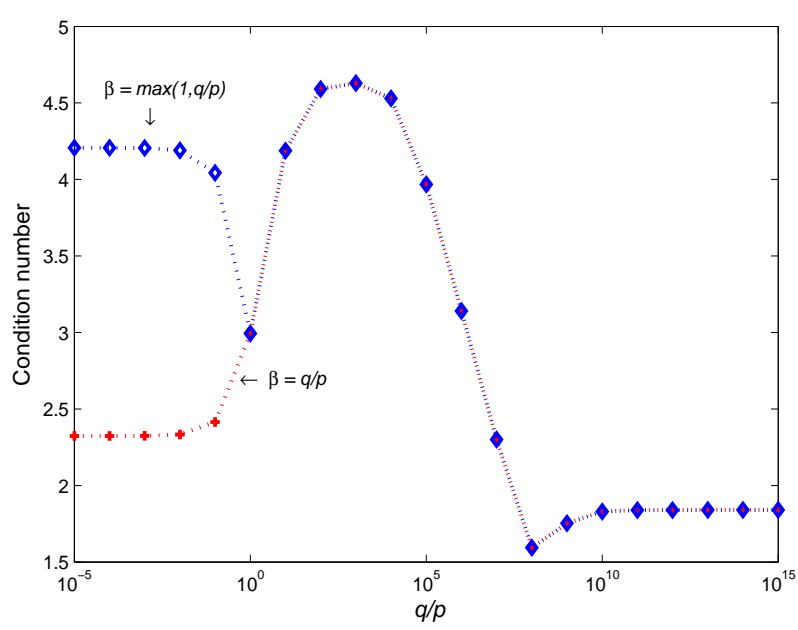

Figure 4. Condition numbers of $\hat{L}_{h}^{-1} \hat{A}_{h N}$, where $\hat{L}_{h}=\hat{B}_{h}+$ BH.

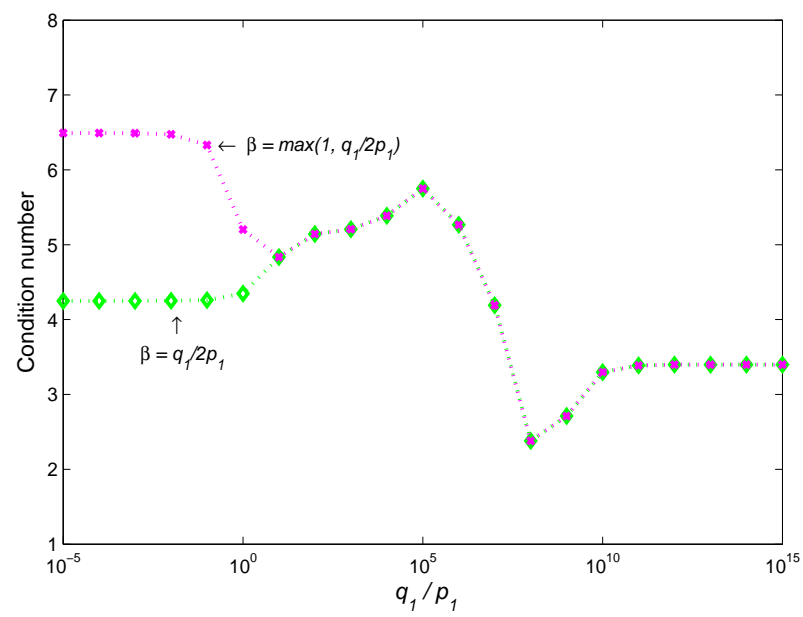

Figure 5. Condition numbers of $\hat{\boldsymbol{L}}_{h}^{-1} \hat{A}_{h N}$, where $\hat{\boldsymbol{L}}_{h}=\hat{B}_{h}+$ $\boldsymbol{\beta H}$.

$$
A u:=-\left(p u^{\prime}\right)^{\prime}+q u \text { in }(-1,1),
$$

we compute the iteration number using PCG preconditioned by $\hat{L}_{h}=\hat{B}_{h}$. Also we compare the developed preconditioners $\hat{L}_{h}$ with the $P_{1}$ finite element preconditioner $\hat{F}_{h}$ (see $[10,12]$ for example) in terms of iteration numbers using PCG.

The computational results are shown in Table 2 with various numbers of elements $E$ and polynomial degrees $N$. While the number of iterations increases as $N$ or $E$ becomes large in case of CG iterations, the PCG preconditioned by $\hat{L}_{h}$ gives relatively small. In particular, it can be predicted that the preconditioning effects become stronger as $N$ or $E$ are made larger. Furthermore we note that the results are comparable to the ones for finite element preconditioner.

\section{Two-Dimensional Case}

In this section the tensor notation is employed. It has the form $A^{y} \otimes B^{x}$ and the superscripts denote the spatial dimension on which it acts. Their order will always be the same and thus we omit them. For the tensor product representation, we refer to [16].

Consider the elliptic operator corresponding to $2 D$ case with zero boundary conditions:

$$
\begin{aligned}
& A u:=-\nabla \cdot p(x, y) \nabla u(x, y)+q(x, y) u(x, y) \\
& \text { in } \Omega=(-1,1) \times(-1,1),
\end{aligned}
$$

where $p \in C^{1}(\bar{\Omega})$ and $q \in C(\bar{\Omega})$ are satisfying $0<\hat{p}_{l} \leq p(x, y) \leq \hat{p}_{u}<\infty, 0 \leq \hat{q}_{l}<q(x, y) \leq \hat{q}_{u}<\infty$.

As in the $1 D$ case, by expanding the variable coefficients $p(x, y)$ and $q(x, y)$ in terms of the basis $\left\{\Phi_{\tilde{\mu}}\right\}_{\tilde{\mu}=1}^{d^{x y}}$ of $\left[\mathcal{P}_{h, N}^{0}\right]^{2}$, two coefficients matrices are ob-

\begin{tabular}{|c|c|c|c|c|c|c|c|c|c|c|c|c|}
\hline$N$ & & 4 & & & 16 & & & 24 & & & 32 & \\
\hline \multirow{2}{*}{$E$} & \multirow{2}{*}{$\mathrm{CG}$} & \multicolumn{2}{|c|}{ PCG } & \multirow{2}{*}{$\mathrm{CG}$} & \multicolumn{2}{|c|}{ PCG } & \multirow{2}{*}{ CG } & \multicolumn{2}{|c|}{ PCG } & \multirow{2}{*}{ CG } & \multicolumn{2}{|c|}{ PCG } \\
\hline & & $\hat{L}_{h}$ & $\hat{F}_{h}$ & & $\hat{L}_{h}$ & $\hat{F}_{h}$ & & $\hat{L}_{h}$ & $\hat{F}_{h}$ & & $\hat{L}_{h}$ & $\hat{F}_{h}$ \\
\hline 1 & 3 & 3 & 3 & 10 & 8 & 8 & 17 & 9 & 9 & 25 & 10 & 10 \\
\hline 2 & 5 & 5 & 5 & 22 & 10 & 10 & 40 & 11 & 11 & 61 & 12 & 12 \\
\hline 4 & 9 & 7 & 7 & 45 & 10 & 10 & 74 & 11 & 12 & 104 & 12 & 12 \\
\hline 8 & 17 & 7 & 8 & 80 & 10 & 11 & 115 & 12 & 12 & 165 & 13 & 13 \\
\hline 16 & 34 & 7 & 8 & 144 & 11 & 11 & 188 & 12 & 12 & 222 & 13 & 13 \\
\hline 32 & 64 & 7 & 8 & 204 & 11 & 11 & 273 & 12 & 13 & 329 & 14 & 14 \\
\hline
\end{tabular}
tained:

Table 2. Iteration numbers in $1 D$ 


$$
\begin{gathered}
\underline{P}:=\operatorname{diag}\left(p_{\tilde{\mu}}:=p_{\mu v}\right), \\
\underline{Q}:=\operatorname{diag}\left(q_{\tilde{\mu}}:=q_{\mu v}\right), \\
\tilde{\mu}=\mu+d^{x}(v-1) .
\end{gathered}
$$

Then the matrix from the spectral element discretization, using one-dimensional matrices $D$ and $W$, becomes

$$
\begin{aligned}
\hat{A}_{h^{2} N^{2}}: & =\left(I \otimes D^{\mathrm{T}}\right) \underline{P}(W \otimes W)(I \otimes D) \\
& +\left(I \otimes M^{\mathrm{T}}\right) \underline{Q}(W \otimes W)(I \otimes M) \\
& +\left(D^{\mathrm{T}} \otimes I\right) \underline{P}(W \otimes W)(D \otimes I) \\
& +\left(M^{\mathrm{T}} \otimes I\right) \underline{Q}(W \otimes W)(M \otimes I),
\end{aligned}
$$

where $M$ is the $1 D$ mass matrix, which becomes the identity for the Lagrange basis $\left\{\Phi_{\tilde{\mu}}\right\}_{\tilde{\mu}=1}^{d^{x y}}$.

From the similar argument in $1 D$ case such as (4.3)(4.4), we have

$$
\begin{aligned}
& \left(\left(\hat{p}_{l}(W \otimes \tilde{K})+\hat{q}_{l}(W \otimes W)\right.\right. \\
& \left.\left.+\hat{p}_{l}(\tilde{K} \otimes W)+\hat{q}_{l}(W \otimes W)\right) V, V\right) \\
& \leq\left(\hat{A}_{h^{2} N^{2}} V, V\right) \leq\left(\left(\hat{p}_{u}(W \otimes \tilde{K})+\hat{q}_{u}(W \otimes W)\right.\right. \\
& \left.\left.\quad+\hat{p}_{u}(\tilde{K} \otimes W)+\hat{q}_{u}(W \otimes W)\right) V, V\right)
\end{aligned}
$$

for any nonzero vector $V$.

In order to describe the preconditioner for the $2 D$ case, with the operator $L_{h^{2}}$ defined in (2.6), we consider a bilinear form $l_{N^{2}}(\cdot, \cdot)$ on $\mathcal{V}_{h, N}^{0} \times \mathcal{V}_{h, N}^{0}$

$$
l_{N^{2}}(u, v):=\sum_{\mu=1 v=1}^{d^{x}} \sum^{d^{y}}\left[L_{h^{2}} u\right]_{\mu, v} v_{\mu, v},
$$

which becomes

$$
l_{N^{2}}(u, v)=\left(\hat{L}_{h^{2}} U, V\right),
$$

where

$$
\begin{aligned}
\hat{L}_{h^{2}}:= & \alpha\left(H^{y} \otimes \hat{B}_{h}^{x}\right)+\beta\left(H^{y} \otimes H^{x}\right) \\
& +\alpha\left(\hat{B}_{h}^{y} \otimes H^{x}\right)+\beta\left(H^{y} \otimes H^{x}\right)
\end{aligned}
$$

and $\hat{B}_{h}^{t}$ is the matrix induced from (2.7) for each $t=x$ or $y$.

Notice that in the proof of Lemma 4.1 it is shown that $(\tilde{K} U, U) \sim\left(\hat{B}_{h} U, U\right)$ is for any nonzero vector $U$. Using this fact and Proposition 3.5, we have the following. have

Theorem 5.1. For any vector $V=\left(v_{1}, \cdots, v_{d^{x y}}\right)^{T}$, we

$$
\left(\hat{A}_{h^{2} N^{2}} V, V\right) \sim\left(\hat{L}_{h^{2}} V, V\right),
$$

where the equivalence are dependent only on $\hat{p}_{u}, \hat{p}_{l}$, $\hat{q}_{u}, \hat{q}_{l}, \alpha$ and $\beta$.
Hence, the eigenvalues of $\hat{L}_{h^{2}}^{-1} \hat{A}_{h^{2} N^{2}}$ are all positive and bounded. The bounds are independent of the mesh sizes $h_{j}^{t}$ and the degrees $N_{k}^{t}$, where $t=x$ or $y$.

Remark 3. This result reveals, as in $1 D$ case, that the upper bound for the condition numbers of the matrix $\hat{L}_{h^{2}}^{-1} \hat{A}_{h^{2} N^{2}}$ is dependent only on the variable coefficients $p(x, y)$ and $q(x, y)$, precisely the condition numbers are bounded above by $\frac{\max \left(\hat{p}_{u}, \hat{q}_{u}\right)}{\min \left(\hat{p}_{l}, \hat{q}_{l}\right)} \frac{\max (\alpha, \beta)}{\min (\alpha, \beta)}$ or $\frac{\max \left(\hat{p}_{u}, \hat{q}_{u}\right)}{\hat{p}_{l}}$.

In the following example, the various eigenvalues and condition numbers of the preconditioned matrix are reported. They are compared with their $P_{1}$ finite element sibling. Also included is a comparison of the iteration counts, for a PCG solver, for the proposed preconditioners.

Example 1. Consider the homogeneous Dirichlet boundary value problem

$$
A u:=-\nabla \cdot(p \nabla u)+q u \text { in } \Omega=(-1,1) \times(-1,1),
$$

where $p(x, y)=x^{2} y^{2}+x^{2}+y^{2}+1$ and $q(x, y)=\cos x \cos y$.

As in $1 D$ case, we consider $\beta=\hat{q} / 4 \hat{p}$ or $\max \{1, \hat{q} / 4 \hat{p}\}$ to have a good preconditioning work, which are shown in Figure 6.

Example 2. For the homogeneous Dirichlet boundary value problem

$$
A u:=-\nabla \cdot \nabla u+10 u \text { in } \Omega=(-1,1) \times(-1,1),
$$

we now compare the developed preconditioners $\hat{L}_{h^{2}}$ with the $P_{1}$ finite element preconditioner $\hat{F}_{h^{2}}$ in terms of iteration numbers using the PCG.

As in the $1 D$ case, we compare the number of PCG iterations for the preconditioners $\hat{L}_{h^{2}}$ and $\hat{F}_{h^{2}}$ which is provided in [12]. In Table 3, we can see that the suggested finite difference preconditioner $\hat{L}_{h^{2}}$ is more

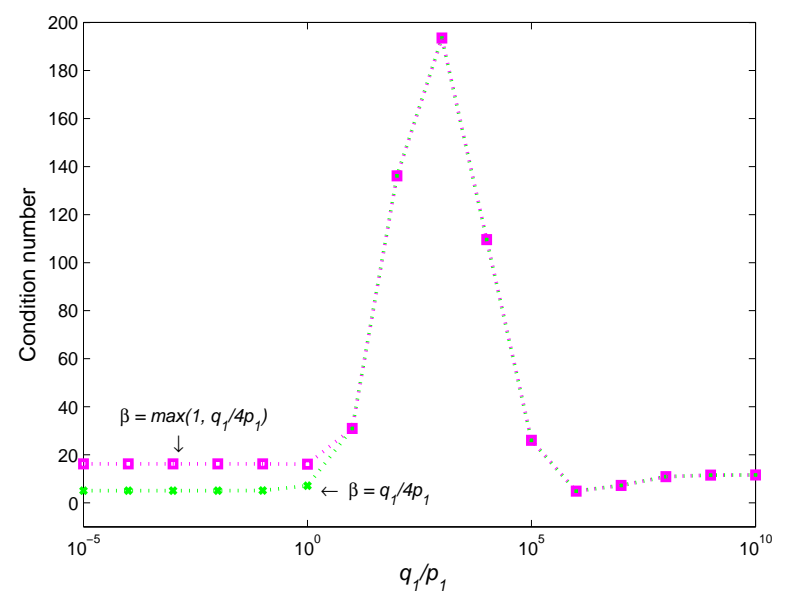

Figure 6. Condition numbers of $\hat{L}_{h^{2}}^{-1} \hat{A}_{h^{2} N^{2}}$. 
Table 3. Iteration numbers in $2 \mathrm{D}$.

\begin{tabular}{|c|c|c|c|c|c|c|c|c|c|}
\hline$N$ & & 4 & & & 8 & & & 12 & \\
\hline \multirow{2}{*}{$E$} & \multirow{2}{*}{ CG } & \multicolumn{2}{|c|}{ PCG } & \multirow{2}{*}{$\mathrm{CG}$} & \multicolumn{2}{|c|}{ PCG } & \multirow{2}{*}{$\mathrm{CG}$} & \multicolumn{2}{|c|}{ PCG } \\
\hline & & $\hat{L}_{h^{2}}$ & $\hat{F}_{h^{2}}$ & & $\hat{L}_{h^{2}}$ & $\hat{F}_{h^{2}}$ & & $\hat{L}_{h^{2}}$ & $\hat{F}_{h^{2}}$ \\
\hline 1 & 4 & 4 & 4 & 10 & 6 & 7 & 15 & 7 & 9 \\
\hline 2 & 11 & 6 & 8 & 24 & 7 & 11 & 38 & 8 & 12 \\
\hline 4 & 16 & 6 & 10 & 29 & 7 & 11 & 46 & 8 & 12 \\
\hline 6 & 19 & 6 & 10 & 31 & 7 & 11 & 48 & 8 & 13 \\
\hline
\end{tabular}

efficient as compared to the $P_{1}$ finite element preconditioner $\hat{F}_{h^{2}}$.

\section{Conclusion}

We have proposed finite differences preconditioners $\hat{L}_{h}$ and $\hat{L}_{h^{2}}$ for spectral element discretizations, and constructed on LGL nodes for uniformly elliptic problem in $n$-dimension, $(n=1,2)$, respectively. The two preconditioners optimality for the corresponding spectral elements problems was demonstrated through the theoretical proofs and the computational results. The burden of the efficiency is now on the multigrid algorithm for solving finite-differences problems and not on $\mathrm{MG}$ for high-order elements.

\section{REFERENCES}

[1] M. O. Deville and E. H. Mund, "Finite Element Preconditioning for Pseudospectral Solutions of Elliptic Problems," SIAM Journal on Scientific and Statistical Computing, Vol. 11, No. 2, 1990, pp. 311-342. doi: $10.1137 / 0911019$

[2] S. D. Kim and S. Parter, "Preconditioning Legendre spectral Collocation Method for Elliptic Partial Differential Equations," SIAM Journal on Numerical Analysis, Vol. 33, No. 6, 1996, pp. 2375-2400. doi:10.1137/S0036142994275998

[3] S. D. Kim and S. Parter, "Preconditioning Chebyshev Spectral Collocation by Finite Difference Operators," SIAM Journal on Numerical Analysis, Vol. 34, No. 3, 1997, pp. 939-958. doi:10.1137/S0036142995285034

[4] S. Parter, "Preconditioning Legendre Spectral Collocation Methods for Elliptic Problems I: Finite Difference Operators," SIAM Journal on Numerical Analysis, Vol. 39, No. 1, 2001, pp. 330-347. doi:10.1137/S0036142999365060

[5] S. Parter, "Preconditioning Legendre Spectral Collocation Methods for Elliptic Problems II: Finite Element Operators," SIAM Journal on Numerical Analysis, Vol. 39, No. 1, 2001, pp. 348-362. doi:10.1137/S0036142999365072

[6] S. Parter and E. Rothman, "Preconditioning Legendre
Spectral Collocation Approximations to Elliptic Problems," SIAM Journal on Numerical Analysis, Vol. 32, No. 2, 1995, pp. 333-385. doi:10.1137/0732015

[7] A. Quarteroni and E. Zampieri, "Finite Element Preconditioning for Legendre Spectral Collocation Approximations to Elliptic Equations and Systems," SIAM Journal on Numerical Analysis, Vol. 29, No. 4, 1992, pp. 917-936. doi: $10.1137 / 0729056$

[8] S. A. Orszag, "Spectral Methods for Problems in Complex Geometries," Journal of Computational Physics, Vol. 37, No. 1, 1980, pp. 70-92. doi:10.1016/0021-9991(80)90005-4

[9] J. Heys, T. Manteuffel, S. McCormick and L. Olson, "Algebraic Multigrid (AMG) for High-Order Finite Elements," Journal of Computational Physics, Vol. 204, No. 2, 2005, pp. 520-532. doi:10.1016/j.jcp.2004.10.021

[10] P. Fischer, “An Overlapping Schwarz Method for Spectral Element Solution of the Incompressible Navier-Stokes equations," Journal of Computational Physics, Vol. 133, No. 1, 1997, pp. 84-101. doi:10.1006/jcph.1997.5651

[11] J. W. Lottes and P. F. Fischer, "Hybrid Multigrid/Schwarz Algorithms for the Spectral Element Method," Journal of Scientific Computing, Vol. 24, No. 1, 2005, pp. 45-78. doi:10.1007/s10915-004-4787-3

[12] S. D. Kim, "Piecewise Bilinear Preconditioning on HighOrder Finite Element Methods," Electronic Transactions on Numerical Analysis, Vol. 26, 2007, pp. 228-242.

[13] S. Kim and S. D. Kim, "Preconditioning on High-Order Element Methods Using Chebyshev-Gauss-Lobatto Nodes," Applied Numerical Mathematics, Vol. 59, No. 2, 2009, pp. 316-333. doi:10.1016/j.apnum.2008.02.007

[14] C. Canuto, M. Y. Hussaini, A. Quarteroni and T. A. Zang, "Spectral Methods: Fundamentals in Single Domains," Springer, New York, 2006.

[15] C. Canuto, M. Y. Hussaini, A. Quarteroni and T. A. Zang, "Spectral Methods: Evolution to Complex Geometries and Applications to Fluid Dynamics," Springer, New York, 2007.

[16] M. O. Deville, P. F. Fischer and E. H. Mund, "High-Order Methods for Incompressible Fluid Flow," In: Cambridge Monographs on Applied and Computational Mathematics, Cambridge University Press, Cambridge, 2002. 